prevention', 'Secondary prevention', Antibiotic, Norfloxacin, Rifaximin, Ciprofloxacin and TMP-SMX. Only those randomised studies were included which evaluated the role of antibiotics in high-risk cirrhosis patients of more than 18 years of age for primary or secondary prophylaxis of spontaneous bacterial peritonitis. Our outcomes were occurrence/recurrence of SBP episode, extra-peritoneal infections, any adverse effects and reduction in mortality. We did random-effects network meta-analysis using a Bayesian approach, and calculated odds ratios (ORs) and 95\% CrI; agents were ranked using rank probabilities.

Results We found total 1211 records in our systematic database search and out of these 17 randomised trials were found eligible for network meta-analysis.

In aggregate group of both primary and secondary SBP, for the outcome of SBP occurrence/recurrence Rifaximin daily, Norfloxacin daily, Rifaximin Norfloxacin daily, Ciprofloxacin intermittent and Trimethoprim-sulfamethoxazole daily were found to be having an odds of $0.050(0.0049-0.21), 0.17$ (0.048-0.41), 0.053(0.0036-0.35), 0.17(0.028-0.76) and 0.16 $(0.027-0.62)$ as compared to that of placebo. The rank probability showed that Rifaximin Norfloxacin daily and Rifaximin daily has a probability of 0.42 and 0.41 respectively for getting ranked as one.

Conclusions CIprofloxacin, Norfloxacin, Trimethoprim-sulfamethoxazole and Rifaximin are useful in prophylaxis of SBP in patients with cirrhosis.

\section{IDDF2019-ABS-0347 PLATELET COUNT AFTER LIVING DONOR LIVER TRANSPLANTATION AS A SURROGATE FOR PORTAL VEIN PRESSURE MONITORING AND PREDICTOR OF GRAFT HYPERPERFUSION SYNDROME: THE '5-67- 8' RULE}

Jeffrey Samuel Co*, CC Wang, CC Yong, CC Lin. Kaohsiung Chang Gung Memorial Hospital, Taiwan

\subsection{6/gutjnl-2019-IDDFabstracts. 16}

Background Thrombocytopenia early after living donor liver transplantation (LDLT) mainly occurs secondary to hepatic/ splenic sequestration, a product of Portal vein pressure (PVP). Portal vein flow (PVF) unlike PVP, is mainly an inflow parameter and does not directly reflect sinusoidal pressure and predict graft dysfunction secondary to hyperperfusion syndrome (HPS). The aim of this study is to determine whether postLDLT platelet count (PC) can reflect PVP and serve as a biomarker to predict HPS.

Methods A total of 757 consecutive adult to adult LDLTs were performed from July 2010 to January 2018 at Kaohsiung Chang Gung Memorial Hospital. After excluding recipients who underwent splenectomy or splenic artery ligation, a total of 690 patients were included. Postoperative liver function, the volume of ascites and graft hemodynamics were recorded on days 1,3,5,7 and 14. Correlation analysis was done using Generalized estimating equations and Receiver operating characteristic analysis (ROC) with Youden's index to determine the optimal cut-off point.

Results A total of 201 patients (29\%) developed HPS in this study. Post LDLT PC significantly correlated to PVF $(p<0.001)$ and HPS $(p<0.001)$. Multiple linear regression analysis showed that PC was a better predictor of HPS when compared to PVF (R2 0.02 vs 0.003 ). PC at postoperative day 5 (POD5) was also a satisfactory biomarker for PVP and predictor of HPS with an AUC of 0.628. Youden's index revealed that the optimal cut-off point for PC was 67,000 per $\mathrm{mm} 3$ with a sensitivity of 0.78 and specificity of 0.59 .

Conclusions Platelet counts within the first 2 weeks after LDLT can serve a surrogate for PVP monitoring and can be used as a guide for further inflow modulation. Furthermore, a PC on POD5 of less than or equal to 67,000 per $\mathrm{mm} 3$ is $80 \%$ sensitive in predicting HPS hence, the ' $5-67$ 8 ' rule.

\section{Basic Gastroenterology}

\begin{tabular}{l|l}
\hline IDDF2019-ABS-0027 & URINARY FORMATE AND GLYCINE ARE \\
& ASSOCIATED WITH TREATMENT RESPONSE \\
& IN PATIENTS TREATED WITH ANTIBIOTICS \\
& FOR POUCHITIS
\end{tabular}

${ }^{1}$ Jonathan Segal ${ }^{*},{ }^{2}$ Magali Sarafian, ${ }^{2}$ Ivan Jose Serrano Contreras, ${ }^{2}$ Alexandros Pechlivanis, ${ }^{2}$ Jerusa Brignardello, ${ }^{2}$ Yih-harn Siaw, ${ }^{2}$ Lucia Braz, ${ }^{2}$ Susan Clark, ${ }^{2}$ Elaine Holmes. ${ }^{1}$ St Mark's Hospital, UK; ${ }^{2}$ Imperial College London, UK

\subsection{6/gutjnl-2019-IDDFabstracts. 17}

Background Restorative proctocolectomy (RPC) is considered the preferred surgical choice for patient with ulcerative colitis (UC) who have failed medical therapy and in some patients with familial adenomatous polyposis (FAP). It has been shown through metabolic profiling of urine that $\mathrm{CD}$ patients have higher levels of formate and lower levels of hippurate and 4-cresol sulfate when compared to healthy controls. To date, extensive metabolic profiling in RPC has yet to be studied.

This study aimed to determine compounds found in urine that are associated with treatment response in patients that have been treated for pouchitis

Methods Patients with pouchitis were recruited from a single centre. Pouchitis was defined using the pouch disease activity index (PDAI) and pouchitis was considered when the score was 37. Response to antibiotics was defined as either a 2 points reduction in PDAI.

Mid-stream morning urine samples were collected. Samples we stored at $-80^{\circ} \mathrm{C}$ until analysis. ${ }^{1} \mathrm{H}$-NMR profile was recorded using the Bruker ${ }^{\circledR}$ Avance III $600 \mathrm{MHz}$ spectrometer, with a Samplejet 96 well autosampler. The full resolution ${ }^{1} \mathrm{H}$ NMR spectra were imported into the SIMCA-P software package, and multivariate data analyses were carried out. Metabolite assignment was performed by comparing chemical shifts, Jres coupling, and peaks multiplicity

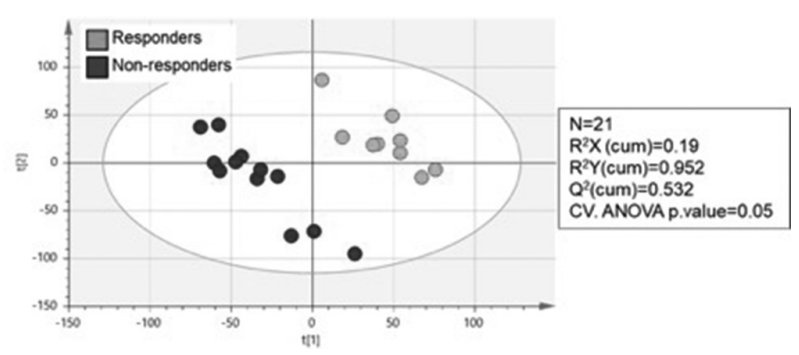

Abstract IDDF2019-ABS-0027 Figure 1 UV PLS DA showing differences between responders vs non-responders 